\title{
A three-term conjugate gradient method with sufficient descent property for unconstrained optimization
}

\author{
Yasushi Narushima, Hiroshi Yabe and John A. Ford
}

(December 10, 2008; Revised October 31, 2009)

\begin{abstract}
Conjugate gradient methods are widely used for solving large-scale unconstrained optimization problems, because they do not need the storage of matrices. In this paper, we propose a general form of three-term conjugate gradient methods which always generate a sufficient descent direction. We give a sufficient condition for the global convergence of the proposed general method. Moreover, we present a specific three-term conjugate gradient method based on the multi-step quasi-Newton method. Finally, some numerical results of the proposed method are given.
\end{abstract}

keyword; Unconstrained optimization, three-term conjugate gradient method, sufficient descent condition, global convergence

\section{Introduction}

In this paper, we deal with conjugate gradient methods for solving the following unconstrained optimization problem:

$$
\text { minimize } f(x) \text {, }
$$

where $f$ is a continuously differentiable function. We denote its gradient $\nabla f$ by $g$. Usually, iterative methods are used for solving unconstrained optimization problems, and they are of the form

$$
x_{k+1}=x_{k}+\alpha_{k} d_{k}
$$

where $x_{k} \in \boldsymbol{R}^{n}$ is the $k$-th approximation to a solution, $\alpha_{k}$ is a positive step size and $d_{k} \in \boldsymbol{R}^{n}$ is a search direction.

In 1952, Hestenes and Stiefel [15] first proposed a conjugate gradient method for solving a linear system of equations with a symmetric positive definite coefficient matrix, 
or equivalently for minimizing a strictly convex quadratic function. Later on, in 1964, Fletcher and Reeves [6] applied the conjugate gradient method to general unconstrained optimization problems. Recently, conjugate gradient methods are paid attention to as iterative methods for solving large-scale unconstrained optimization problems, because they do not need the storage of matrices. The search direction of conjugate gradient methods is defined by the following:

$$
d_{k}= \begin{cases}-g_{k}, & \text { for } \quad k=0 \\ -g_{k}+\beta_{k} d_{k-1}, & \text { for } \quad k \geq 1\end{cases}
$$

where $g_{k}$ denotes $\nabla f\left(x_{k}\right)$ and $\beta_{k} \in \boldsymbol{R}$ is a parameter that characterizes the method. It is known that choices of $\beta_{k}$ affect numerical performance of the method, and hence many researchers studied choices of $\beta_{k}$. Well-known formulas for $\beta_{k}$ are the HestenesStiefel (HS) [15, 16], Fletcher-Reeves (FR) [6], Polak-Ribière (PR) [16], Polak-Ribière Plus (PR+) [10], and Dai-Yuan (DY) [4] formulas, which are respectively given by

$$
\begin{gathered}
\beta_{k}^{H S}=\frac{g_{k}^{T} y_{k-1}}{d_{k-1}^{T} y_{k-1}}, \quad \beta_{k}^{F R}=\frac{\left\|g_{k}\right\|^{2}}{\left\|g_{k-1}\right\|^{2}}, \\
\beta_{k}^{P R}=\frac{g_{k}^{T} y_{k-1}}{\left\|g_{k-1}\right\|^{2}}, \quad \beta_{k}^{P R+}=\max \left\{\frac{g_{k}^{T} y_{k-1}}{\left\|g_{k-1}\right\|^{2}}, 0\right\}, \quad \beta_{k}^{D Y}=\frac{\left\|g_{k}\right\|^{2}}{d_{k-1}^{T} y_{k-1}},
\end{gathered}
$$

where $y_{k-1}$ is defined by

$$
y_{k-1}=g_{k}-g_{k-1}
$$

and $\|\cdot\|$ denotes the $\ell_{2}$ norm. Furthermore, we define

$$
s_{k-1}=x_{k}-x_{k-1},
$$

which is used in the subsequent sections. Note that these formulas for $\beta_{k}$ are equivalent each other if the objective function is a strictly convex quadratic function and $\alpha_{k}$ is the one dimensional minimizer. There are many researches on convergence properties of these methods (see $[13,16]$, for example).

For this decade, many other conjugate gradient methods are proposed and these are classified by two classes. The first approach makes use of the second-order information of the objective function to accelerate conjugate gradient methods. Dai and Liao [3] proposed a conjugate gradient method based on the secant condition and proved its global convergence property. Later some researchers proposed its variants based on other secant conditions, and they proved global convergence properties of their proposed methods $[9,18,22]$. Although these methods are effective for solving large-scale unconstrained optimization problems in our numerical experiments, they do not necessarily satisfy the descent condition (i.e. $g_{k}^{T} d_{k}<0$ for all $k$ ). The second approach aims to generate a descent search direction. Dai and Yuan [4] proposed a conjugate gradient method which generates descent search directions under the Wolfe conditions. Later Yabe and Sakaiwa [17] gave 
its variant which also generates descent search directions. Independently of Dai-Yuan's research, Hager and Zhang [12] proposed a conjugate gradient method which generates the descent search direction under the Wolfe conditions. However, these methods depend on line searches to satisfy the descent condition. Conjugate gradient methods which have the both characteristics of the two approaches above have not been proposed.

More recently, Zhang, Zhou and Li. [19-21] proposed three-term conjugate gradient methods which always satisfy the sufficient descent condition:

$$
g_{k}^{T} d_{k} \leq-\bar{c}\left\|g_{k}\right\|^{2} \quad \text { for all } k
$$

for a positive constant $\bar{c}$, independently of line searches. They proposed the modified FR method [20] defined by

$$
d_{k}=-\bar{\theta}_{k} g_{k}+\beta^{F R} d_{k-1}
$$

where $\bar{\theta}_{k}=d_{k-1}^{T} y_{k-1} /\left\|g_{k-1}\right\|^{2}$. Since this search direction satisfies $g_{k}^{T} d_{k}=-\left\|g_{k}\right\|^{2}$ for all $k$, it can be rewritten by the three-term form:

$$
d_{k}=-g_{k}+\beta^{F R} d_{k-1}-\theta_{k}^{(1)} g_{k}
$$

where $\theta_{k}^{(1)}=g_{k}^{T} d_{k-1} /\left\|g_{k-1}\right\|^{2}$. They also proposed the modified PR method [19] and the modified HS method [21], which are respectively given by

$$
\begin{aligned}
& d_{k}=-g_{k}+\beta^{P R} d_{k-1}-\theta_{k}^{(2)} y_{k-1}, \\
& d_{k}=-g_{k}+\beta^{H S} d_{k-1}-\theta_{k}^{(3)} y_{k-1},
\end{aligned}
$$

where $\theta_{k}^{(2)}=g_{k}^{T} d_{k-1} /\left\|g_{k-1}\right\|^{2}$ and $\theta_{k}^{(3)}=g_{k}^{T} d_{k-1} / d_{k-1}^{T} y_{k-1}$. Cheng [2] gave another modified PR method:

$$
d_{k}=-g_{k}+\beta_{k}^{P R}\left(I-\frac{g_{k} g_{k}^{T}}{g_{k}^{T} g_{k}}\right) d_{k-1}=-g_{k}+\beta_{k}^{P R} d_{k-1}-\beta_{k}^{P R} \frac{g_{k}^{T} d_{k-1}}{g_{k}^{T} g_{k}} g_{k} .
$$

They showed their global convergence properties under appropriate line searches. We note that these methods always satisfy $g_{k}^{T} d_{k}=-\left\|g_{k}\right\|^{2}<0$ for all $k$, which implies the sufficient descent condition with $\bar{c}=1$.

In this paper, by modifying (1.1), we propose a general form of three-term conjugate gradient methods which always satisfy (1.3), independently of choices of $\beta_{k}$ and line searches. Moreover, we establish its global convergence property. The present paper is organized as follows. In Section 2, we construct a general form of three-term conjugate gradient methods which satisfy (1.3), and give a sufficient condition for its global convergence. In Section 3, we propose a specific three-term conjugate gradient method based on the multi-step quasi-Newton method, and prove its global convergence by using the result of Section 2. Finally, in Section 4, some numerical experiments are presented. 


\section{Three-term conjugate gradient method and its con- vergence property}

In this section, we consider a three-term conjugate gradient method to obtain a descent search direction. Section 2.1 presents a general form of three-term conjugate gradient methods and Section 2.2 shows its global convergence property.

\subsection{Three-term conjugate gradient method}

We propose a new three-term conjugate gradient method of the form:

$$
\begin{gathered}
x_{k+1}=x_{k}+\alpha_{k} d_{k}, \\
d_{k}= \begin{cases}-g_{k} & k=0, \\
-g_{k}+\beta_{k}\left(g_{k}^{T} p_{k}\right)^{\dagger}\left\{\left(g_{k}^{T} p_{k}\right) d_{k-1}-\left(g_{k}^{T} d_{k-1}\right) p_{k}\right\} & k \geq 1,\end{cases}
\end{gathered}
$$

where $\beta_{k} \in \boldsymbol{R}$ is a parameter, $p_{k} \in \boldsymbol{R}^{n}$ is any vector and

$$
a^{\dagger}= \begin{cases}\frac{1}{a} & a \neq 0 \\ 0 & a=0 .\end{cases}
$$

We emphasize that the method (2.1)-(2.2) always satisfies

$$
g_{k}^{T} d_{k}=-\left\|g_{k}\right\|^{2}
$$

independently of choices of $p_{k}$ and line searches. It means that the sufficient descent condition (1.3) holds with $\bar{c}=1$.

Note that $(2.2)$ can be rewritten by

$$
d_{k}= \begin{cases}-g_{k} & \text { if } k=0 \text { or } g_{k}^{T} p_{k}=0, \\ -g_{k}+\beta_{k} d_{k-1}-\beta_{k} \frac{g_{k}^{T} d_{k-1}}{g_{k}^{T} p_{k}} p_{k} & \text { otherwise. }\end{cases}
$$

Accordingly, if $g_{k}^{T} p_{k} \neq 0$ is satisfied, the form (2.2) becomes

$$
d_{k}=-g_{k}+\beta_{k}\left(I-\frac{p_{k} g_{k}^{T}}{g_{k}^{T} p_{k}}\right) d_{k-1} .
$$

The matrix $\left(I-p_{k} g_{k}^{T} / g_{k}^{T} p_{k}\right)$ is a projection matrix into the orthogonal complement of $\operatorname{Span}\left\{g_{k}\right\}$ along $\operatorname{Span}\left\{p_{k}\right\}$. Especially, if we choose $p_{k}=g_{k}$, then $\left(I-g_{k} g_{k}^{T} /\left\|g_{k}\right\|^{2}\right)$ is an orthogonal projection matrix.

If we use the exact line search and $p_{k}$ such that $g_{k}^{T} p_{k} \neq 0$, then our method (2.4) becomes the nonlinear conjugate gradient method (1.1). The most simple choices are 
$p_{k}=g_{k}$ and $p_{k}=y_{k-1}$. On the other hand, if we choose $p_{k}=d_{k-1}$, then (2.2) implies $d_{k}=-g_{k}$ for all $k$.

We should note that the present method includes the three-term conjugate gradient methods proposed by Zhang et al. [19-21]. The method (2.1)-(2.2) with $\beta_{k}=\beta_{k}^{F R}$ and $p_{k}=g_{k}$ becomes the method by [20] (see (1.4)), and, if $g_{k}^{T} y_{k-1} \neq 0$, the method (2.1)-(2.2) with $\beta_{k}=\beta_{k}^{P R}$ and $p_{k}=y_{k-1}$ becomes the method by [19] (see (1.5)). If $g_{k}^{T} y_{k-1} \neq 0$, the method (2.1)-(2.2) with $\beta_{k}=\beta_{k}^{H S}$ and $p_{k}=y_{k-1}$ becomes the method by [21] (see (1.6)). In addition, the method (2.1)-(2.2) with $\beta_{k}=\beta_{k}^{P R}$ and $p_{k}=g_{k}$ becomes the method by [2] (see (1.7)).

\subsection{Convergence analysis}

In order to establish the global convergence property, we make the following standard assumptions for the objective function.

\section{Assumption 2.1.}

1. The level set $\mathcal{L}=\left\{x \mid f(x) \leq f\left(x_{0}\right)\right\}$ at $x_{0}$ is bounded, namely, there exists a constant $\widehat{a}>0$ such that

$$
\|x\| \leq \widehat{a} \quad \text { for all } \quad x \in \mathcal{L}
$$

2. In some neighborhood $\mathcal{N}$ of $\mathcal{L}, f$ is continuously differentiable, and its gradient is Lipschitz continuous with Lipschitz constant $L>0$, i.e.

$$
\|g(u)-g(v)\| \leq L\|u-v\| \quad \text { for all } u, v \in \mathcal{N} .
$$

Assumption 2.1 implies that there exists a positive constant $\widehat{\gamma}$ such that

$$
\|g(x)\| \leq \widehat{\gamma}, \quad \text { for all } \quad x \in \mathcal{L}
$$

In the line search, we require $\alpha_{k}$ to satisfy the Wolfe conditions:

$$
\begin{aligned}
f\left(x_{k}\right)-f\left(x_{k}+\alpha_{k} d_{k}\right) & \geq-\delta \alpha_{k} g_{k}^{T} d_{k}, \\
g\left(x_{k}+\alpha_{k} d_{k}\right)^{T} d_{k} & \geq \sigma g_{k}^{T} d_{k}
\end{aligned}
$$

where $0<\delta<\sigma<1$, or the strong Wolfe conditions: (2.8) and

$$
\left|g\left(x_{k}+\alpha_{k} d_{k}\right)^{T} d_{k}\right| \leq \sigma\left|g_{k}^{T} d_{k}\right|
$$

where $0<\delta<\sigma<1$.

In the rest of this section, we assume $g_{k} \neq 0$ for all $k$, otherwise a stationary point has been found.

Under Assumption 2.1, we have the following well-known lemma which was proved by Zoutendijk (see [16]). The following lemma is the result for general iterative methods with the Wolfe condition (2.8) and (2.9). 
Lemma 2.1. Suppose that Assumption 2.1 is satisfied. Consider any method in the form (2.1), where $d_{k}$ is a descent search direction and $\alpha_{k}$ satisfies the Wolfe conditions (2.8) and (2.9). Then

$$
\sum_{k=0}^{\infty} \frac{\left(g_{k}^{T} d_{k}\right)^{2}}{\left\|d_{k}\right\|^{2}}<\infty
$$

Using Lemma 2.1, we have the following lemma, which is useful in showing the global convergence of our method.

Lemma 2.2. Suppose that Assumption 2.1 is satisfied. Consider the method (2.1)-(2.2), where $\alpha_{k}$ satisfies the Wolfe conditions (2.8) and (2.9). If

$$
\sum_{k=0}^{\infty} \frac{1}{\left\|d_{k}\right\|^{2}}=\infty
$$

holds, then the following holds:

$$
\liminf _{k \rightarrow \infty}\left\|g_{k}\right\|=0
$$

Proof. If (2.12) is not true, there exists a constant $\varepsilon>0$ such that

$$
\left\|g_{k}\right\| \geq \varepsilon
$$

for all $k$. Therefore from $(2.3)$ and (2.11), we have

$$
\sum_{k=0}^{\infty} \frac{\varepsilon^{4}}{\left\|d_{k}\right\|^{2}} \leq \sum_{k=0}^{\infty} \frac{\left\|g_{k}\right\|^{4}}{\left\|d_{k}\right\|^{2}}=\sum_{k=0}^{\infty} \frac{\left(g_{k}^{T} d_{k}\right)^{2}}{\left\|d_{k}\right\|^{2}}=\infty
$$

Since this contradicts Lemma 2.1, the proof is complete.

Now we consider a sufficient condition to establish the global convergence property of the method (2.1)-(2.2). First, we estimate the norm of the search direction of the proposed method. If $g_{k}^{T} p_{k}=0$, the following relation

$$
\left\|d_{k}\right\|=\left\|g_{k}\right\|
$$

holds. Otherwise, by squaring both sides of (2.5), we have from the orthogonality of $g_{k}$ and $\left(I-p_{k} g_{k}^{T} / g_{k}^{T} p_{k}\right) d_{k-1}$

$$
\begin{aligned}
\left\|d_{k}\right\|^{2} & =\left\|-g_{k}+\beta_{k}\left(I-\frac{p_{k} g_{k}^{T}}{g_{k}^{T} p_{k}}\right) d_{k-1}\right\|^{2} \\
& =\beta_{k}^{2}\left\|\left(I-\frac{p_{k} g_{k}^{T}}{g_{k}^{T} p_{k}}\right) d_{k-1}\right\|^{2}+\left\|g_{k}\right\|^{2},
\end{aligned}
$$


and hence it follows from $\left\|I-\frac{p_{k} g_{k}^{T}}{g_{k}^{T} p_{k}}\right\|=\frac{\left\|g_{k}\right\|\left\|p_{k}\right\|}{\left|g_{k}^{T} p_{k}\right|}$ that

$$
\left\|d_{k}\right\|^{2} \leq \beta_{k}^{2}\left(\frac{\left\|g_{k}\right\|\left\|p_{k}\right\|}{\left|g_{k}^{T} p_{k}\right|}\right)^{2}\left\|d_{k-1}\right\|^{2}+\left\|g_{k}\right\|^{2} .
$$

Therefore, by defining

$$
\psi_{k}=\beta_{k}\left\|g_{k}\right\|\left\|p_{k}\right\|\left(g_{k}^{T} p_{k}\right)^{\dagger}
$$

relations (2.13) and (2.14) yield

$$
\left\|d_{k}\right\|^{2} \leq \psi_{k}^{2}\left\|d_{k-1}\right\|^{2}+\left\|g_{k}\right\|^{2}
$$

for all $k$.

For standard conjugate gradient methods, Gilbert and Nocedal [10] derived Property (*), which shows that $\beta_{k}$ will be small when the step $s_{k-1}$ is small (see also Dai and Liao [3]). The following property corresponds with Property $(*)$ except for using $\psi_{k}$ instead of $\beta_{k}$.

Property A. Consider the method (2.1)-(2.2). Assume that there exists a positive constant $\varepsilon$ such that $\varepsilon \leq\left\|g_{k}\right\|$ holds for all $k$. Then we say that the method has Property $A$ if there exist constants $b>1$ and $\xi>0$ such that for all $k$ :

$$
\left|\psi_{k}\right| \leq b
$$

and

$$
\left\|s_{k-1}\right\| \leq \xi \Longrightarrow\left|\psi_{k}\right| \leq \frac{1}{b}
$$

We note that (2.17) implies that if there exists a positive constant $\varepsilon$ such that $\varepsilon \leq\left\|g_{k}\right\|$ for all $k$, then

$$
\left|\beta_{k}\right|\left\|p_{k}\right\|\left|g_{k}^{T} p_{k}\right|^{\dagger} \leq c
$$

holds with $c=b / \varepsilon$.

The next lemma corresponds to Lemma 3.4 in Dai and Liao [3].

Lemma 2.3. Suppose that Assumption 2.1 is satisfied. Consider the method (2.1)-(2.2), where $\alpha_{k}$ satisfies the strong Wolfe conditions (2.8) and (2.10). Assume that there exists a positive constant $\varepsilon$ such that the following relation holds $\varepsilon \leq\left\|g_{k}\right\|$ holds for all $k$. If the method has Property $A$ and $\beta_{k} \geq 0$ holds, then $d_{k} \neq 0$ and the following relation holds

$$
\sum_{k=0}^{\infty}\left\|u_{k}-u_{k-1}\right\|^{2}<\infty
$$

where $u_{k}=d_{k} /\left\|d_{k}\right\|$. 
Proof. Since $d_{k} \neq 0$ follows from (2.3) and $\varepsilon \leq\left\|g_{k}\right\|$, the vector $u_{k}$ is well-defined. Using Lemma 2.2 and $\varepsilon \leq\left\|g_{k}\right\|$, we have

$$
\sum_{k=0}^{\infty} \frac{1}{\left\|d_{k}\right\|^{2}}<\infty
$$

By defining

$$
v_{k}=-\left(g_{k}+\beta_{k}\left(g_{k}^{T} p_{k}\right)^{\dagger}\left(g_{k}^{T} d_{k-1}\right) p_{k}\right) \frac{1}{\left\|d_{k}\right\|} \quad \text { and } \quad \eta_{k}=\beta_{k}\left(g_{k}^{T} p_{k}\right)^{\dagger}\left(g_{k}^{T} p_{k}\right) \frac{\left\|d_{k-1}\right\|}{\left\|d_{k}\right\|},
$$

equation (2.2) is written as

$$
u_{k}=v_{k}+\eta_{k} u_{k-1}
$$

Then we have from the fact that $\left\|u_{k}\right\|=\left\|u_{k-1}\right\|=1$,

$$
\left\|v_{k}\right\|=\left\|u_{k}-\eta_{k} u_{k-1}\right\|=\left\|\eta_{k} u_{k}-u_{k-1}\right\| .
$$

It follows from $\beta_{k} \geq 0$ and (2.21) that

$$
\begin{aligned}
\left\|u_{k}-u_{k-1}\right\| & \leq\left(1+\eta_{k}\right)\left\|u_{k}-u_{k-1}\right\| \\
& =\left\|u_{k}-\eta_{k} u_{k-1}+\eta_{k} u_{k}-u_{k-1}\right\| \\
& \leq\left\|u_{k}-\eta_{k} u_{k-1}\right\|+\left\|\eta_{k} u_{k}-u_{k-1}\right\| \\
& =2\left\|v_{k}\right\| .
\end{aligned}
$$

From (2.19), we have

$$
\beta_{k}\left|g_{k}^{T} p_{k}\right|^{\dagger}\left\|p_{k}\right\| \leq c
$$

for all $k$. Therefore by (2.10), (2.3), (2.7) and (2.19), we have

$$
\begin{aligned}
\beta_{k}\left|g_{k}^{T} d_{k-1}\right|\left|g_{k}^{T} p_{k}\right|^{\dagger}\left\|p_{k}\right\| & \leq \sigma \beta_{k}\left|g_{k-1}^{T} d_{k-1}\left\|\left.g_{k}^{T} p_{k}\right|^{\dagger}\right\| p_{k} \|\right. \\
& =\sigma \beta_{k}\left|g_{k}^{T} p_{k}\right|^{\dagger}\left\|p_{k}\right\|\left\|g_{k-1}\right\|^{2} \\
& \leq \sigma c \widehat{\gamma}^{2} .
\end{aligned}
$$

Thus (2.22), (2.7) and (2.20) yield

$$
\begin{aligned}
\sum_{k=0}^{\infty}\left\|u_{k}-u_{k-1}\right\|^{2} & \leq 4 \sum_{k=0}^{\infty}\left\|v_{k}\right\|^{2} \\
& \leq 4 \sum_{k=0}^{\infty}\left(\left\|g_{k}\right\|+\beta_{k}\left|g_{k}^{T} d_{k-1}\left\|\left.g_{k}^{T} p_{k}\right|^{\dagger}\right\| p_{k} \|\right)^{2} \cdot \frac{1}{\left\|d_{k}\right\|^{2}}\right. \\
& \leq 4\left(\widehat{\gamma}+\sigma \widehat{\gamma}^{2} c\right)^{2} \sum_{k=0}^{\infty} \frac{1}{\left\|d_{k}\right\|^{2}} \\
& <\infty .
\end{aligned}
$$


Therefore the lemma is proved.

Let $\boldsymbol{N}$ denote the set of all positive integers. For $\lambda>0$ and a positive integer $\Delta$, we define the set of indices:

$$
\mathcal{K}_{k, \Delta}^{\lambda}:=\left\{i \in N \mid k \leq i \leq k+\Delta-1,\left\|s_{i-1}\right\|>\lambda\right\}
$$

Let $\left|\mathcal{K}_{k, \Delta}^{\lambda}\right|$ denote the number of elements in $\mathcal{K}_{k, \Delta}^{\lambda}$. The following lemma shows that if the gradients are bounded away from zero and (2.17)-(2.18) hold, then a certain fraction of the steps cannot be too small. This lemma corresponds to [3, Lemma 3.5] and [10, Lemma 4.2].

Lemma 2.4. Suppose that all assumptions of Lemma 2.3 hold. If the method has Property A, then there exists $\lambda>0$ such that, for any $\Delta \in \boldsymbol{N}$ and any index $k_{0}$, there is an index $\widehat{k} \geq k_{0}$ such that

$$
\left|\mathcal{K}_{\widehat{k}, \Delta}^{\lambda}\right|>\frac{\Delta}{2}
$$

Proof. We prove this lemma by contradiction. Assume that for any $\lambda>0$, there exist $\Delta \in \boldsymbol{N}$ and $k_{0}$ such that

$$
\left|\mathcal{K}_{k, \Delta}^{\lambda}\right| \leq \frac{\Delta}{2}
$$

for all $k \geq k_{0}$. Let $b>1$ and $\xi>0$ be given in Property A. For $\lambda=\xi$, we choose $\Delta$ and $k_{0}$ such that (2.23) holds. Then from (2.17), (2.18) and (2.23), we have

$$
\prod_{k=k_{0}+i \Delta+1}^{k_{0}+(i+1) \Delta}\left|\psi_{k}\right|=\prod_{k \in \mathcal{K}_{k^{\prime}, \Delta}^{\lambda}}\left|\psi_{k}\right| \prod_{k \notin \mathcal{K}_{k^{\prime}, \Delta}^{\lambda}}\left|\psi_{k}\right| \leq b^{\Delta / 2}\left(\frac{1}{b}\right)^{\Delta / 2}=1 \quad \text { for any } i \geq 0
$$

where $k^{\prime}=k_{0}+i \Delta+1$. If $\psi_{k}=0$ holds, then the search direction becomes $d_{k}=$ $-g_{k}$. Therefore, if $\psi_{k}$ equals zero infinitely many times, the search direction becomes the steepest descent direction infinitely many times, which implies that $\liminf _{k \rightarrow \infty}\left\|g_{k}\right\|=0$. Otherwise, we have $\psi_{k} \neq 0$ for $k$ sufficiently large. Therefore we assume without loss of generality that

$$
\psi_{k} \neq 0
$$

for all $k \geq 1$. It follows from (2.24) that

$$
\prod_{j=2}^{k_{0}+i \Delta}\left|\psi_{j}\right|=\left(\prod_{j=2}^{k_{0}}\left|\psi_{j}\right|\right) \cdot\left(\prod_{j=k_{0}+1}^{k_{0}+\Delta}\left|\psi_{j}\right|\right) \ldots\left(\prod_{j=k_{0}+(i-1) \Delta+1}^{k_{0}+i \Delta}\left|\psi_{j}\right|\right) \leq \prod_{j=2}^{k_{0}}\left|\psi_{j}\right| \quad \text { for any } i \geq 0
$$

which implies by $(2.25)$

$$
\prod_{j=2}^{k_{0}+i \Delta} \psi_{j}^{-2} \geq \prod_{j=2}^{k_{0}} \psi_{j}^{-2} \quad \text { for any } i \geq 0
$$


By summing (2.26), we have

$$
\sum_{k=2}^{\infty} \prod_{j=2}^{k} \psi_{j}^{-2} \geq \sum_{i=0}^{\infty} \prod_{j=2}^{k_{0}+i \Delta} \psi_{j}^{-2} \geq \sum_{i=0}^{\infty} \prod_{j=2}^{k_{0}} \psi_{j}^{-2}=\infty .
$$

From Lemma 2.1 and the assumption $0<\varepsilon \leq\left\|g_{k}\right\|$, we have

$$
\sum_{k=0}^{\infty} \frac{\left(g_{k}^{T} d_{k}\right)^{2}}{\left\|d_{k}\right\|^{2}\left\|g_{k}\right\|^{2}} \leq \sum_{k=0}^{\infty} \frac{\left(g_{k}^{T} d_{k}\right)^{2}}{\varepsilon^{2}\left\|d_{k}\right\|^{2}}<\infty
$$

Thus there exist a integer $j_{0}$ and a constant $c_{2}>0$ such that

$$
\prod_{j=j_{0}}^{k}\left(1-\frac{\left(g_{j}^{T} d_{j}\right)^{2}}{\left\|g_{j}\right\|^{2}\left\|d_{j}\right\|^{2}}\right) \geq c_{2}
$$

holds for any $k \geq j_{0}$. On the other hand, (2.16) and (2.3) yield

$$
\left\|d_{k}\right\|^{2} \leq \psi_{k}^{2}\left\|d_{k-1}\right\|^{2}+\left\|g_{k}\right\|^{2}=\psi_{k}^{2}\left\|d_{k-1}\right\|^{2}+\frac{\left(g_{k}^{T} d_{k}\right)^{2}}{\left\|g_{k}\right\|^{2}}
$$

and hence it follows from (2.28) that

$$
\begin{aligned}
\left\|d_{k}\right\|^{2} & \leq\left(1-\frac{\left(g_{k}^{T} d_{k}\right)^{2}}{\left\|g_{k}\right\|^{2}\left\|d_{k}\right\|^{2}}\right)^{-1} \psi_{k}^{2}\left\|d_{k-1}\right\|^{2} \\
& \leq \cdots \\
& \leq \prod_{j=j_{0}}^{k}\left(1-\frac{\left(g_{j}^{T} d_{j}\right)^{2}}{\left\|g_{j}\right\|^{2}\left\|d_{j}\right\|^{2}}\right)^{-1}\left(\prod_{j=j_{0}}^{k} \psi_{j}^{2}\right)\left\|d_{j_{0}-1}\right\|^{2} \\
& \leq \frac{\left\|d_{j_{0}-1}\right\|^{2}}{c_{2}}\left(\prod_{j=2}^{j_{0}-1} \psi_{j}^{-2}\right)\left(\prod_{j=2}^{k} \psi_{j}^{2}\right) \\
& \leq c_{3} \prod_{j=2}^{k} \psi_{j}^{2}
\end{aligned}
$$

for all $k \geq j_{0}$, where $c_{3}=\frac{\left\|d_{j_{0}-1}\right\|^{2}}{c_{2}} \prod_{j=2}^{j_{0}-1} \psi_{j}^{-2}$. Note that $c_{3}$ is a positive constant, because $j_{0}$ is a fixed integer in (2.28). Therefore, we get by $(2.27)$

$$
\sum_{k=j_{0}}^{\infty} \frac{1}{\left\|d_{k}\right\|^{2}} \geq \frac{1}{c_{3}} \sum_{k=j_{0}}^{\infty} \prod_{j=2}^{k} \psi_{j}^{-2}=\infty
$$

It follows from Lemma 2.2 that $\liminf _{k \rightarrow \infty}\left\|g_{k}\right\|=0$ holds. Since this contradicts the assumption $0<\varepsilon \leq\left\|g_{k}\right\|$, we obtain the desired result.

Now we can give a sufficient condition for the global convergence of the method (2.1)(2.2) by using Lemmas 2.3 and 2.4 and Property A. This theorem corresponds to Theorem 3.6 in [3] and the proof is exactly same as that of Theorem 3.6, but we write it for the readability. 
Theorem 2.1. Consider the method (2.1)-(2.2) that satisfies the following conditions:

(C1) $\beta_{k} \geq 0$ for all $k$,

(C2) Property A holds.

Assume that $\alpha_{k}$ satisfies the strong Wolfe conditions (2.8) and (2.10). If Assumption 2.1 holds, then the method converges in the sense that $\liminf _{k \rightarrow \infty}\left\|g_{k}\right\|=0$.

Proof. Since we prove this theorem by contradiction, we assume that there exists $\varepsilon$ such that $0<\varepsilon \leq\left\|g_{k}\right\|$ holds for all $k$. Then Lemmas 2.3 and 2.4 hold. From the definition of $u_{k}$, we have for any $l$ and $k$ with $l \geq k$,

$$
\begin{aligned}
x_{l}-x_{k-1} & =\sum_{i=k}^{l}\left\|s_{i-1}\right\| u_{i-1} \\
& =\sum_{i=k}^{l}\left\|s_{i-1}\right\| u_{k-1}+\sum_{i=k}^{l}\left\|s_{i-1}\right\|\left(u_{i-1}-u_{k-1}\right) .
\end{aligned}
$$

It follows from this relation, the fact $\left\|u_{k-1}\right\|=1$ and (2.6) that

$$
\begin{aligned}
\sum_{i=k}^{l}\left\|s_{i-1}\right\| & \leq\left\|x_{l}-x_{k-1}\right\|+\sum_{i=k}^{l}\left\|s_{i-1}\right\|\left\|u_{i-1}-u_{k-1}\right\| \\
& \leq 2 \widehat{a}+\sum_{i=k}^{l}\left\|s_{i-1}\right\|\left\|u_{i-1}-u_{k-1}\right\|,
\end{aligned}
$$

which implies that

$$
2 \widehat{a} \geq \sum_{i=k}^{l}\left\|s_{i-1}\right\|\left(1-\left\|u_{i-1}-u_{k-1}\right\|\right) .
$$

Let $\lambda>0$ be given by Lemma 2.4 and define $\Delta=\lceil 8 \widehat{a} / \lambda\rceil$ to be the smallest integer not less than $8 \widehat{a} / \lambda$. By Lemma 2.3, we can find an index $k_{0}$ such that

$$
\sum_{i=k_{0}}^{\infty}\left\|u_{i}-u_{i-1}\right\|^{2} \leq \frac{1}{4 \Delta} .
$$

For $\Delta$ and $k_{0}$ defined above, Lemma 2.4 gives an index $k \geq k_{0}$ such that

$$
\left|\mathcal{K}_{k, \Delta}^{\lambda}\right|>\frac{\Delta}{2}
$$

By (2.30) and the fact that $\|v\|_{1} \leq \sqrt{n}\|v\|$ for any vector $v \in \boldsymbol{R}^{n}$, we have

$$
\begin{aligned}
\left\|u_{i}-u_{k-1}\right\| & \leq \sum_{j=k}^{i}\left\|u_{j}-u_{j-1}\right\| \\
& \leq(i-k+1)^{1 / 2}\left(\sum_{j=k}^{i}\left\|u_{j}-u_{j-1}\right\|^{2}\right)^{1 / 2} \\
& \leq \Delta^{1 / 2}\left(\frac{1}{4 \Delta}\right)^{1 / 2}=\frac{1}{2}
\end{aligned}
$$


for any $i(k \leq i \leq k+\Delta-1)$. Therefore it follows from (2.29) with $l=k+\Delta-1$, the definition of $\mathcal{K}_{k, \Delta}^{\lambda}$ and (2.31) that

$$
2 \widehat{a} \geq \frac{1}{2} \sum_{i=k}^{k+\Delta-1}\left\|s_{i-1}\right\|>\frac{\lambda}{2}\left|\mathcal{K}_{k, \Delta}^{\lambda}\right|>\frac{\lambda \Delta}{4} .
$$

Thus we get $\Delta<8 \widehat{a} / \lambda$, which contradicts the definition of $\Delta$. Therefore, the theorem is true.

Theorem 2.1 plays an important role to establish global convergence properties of various kinds of three-term conjugate gradient methods. For instance, we obtain the following convergence results as a corollary of Theorem 2.1 .

Corollary 2.1. Suppose that Assumption 2.1 is satisfied. Consider the method (2.1)(2.2), where $\alpha_{k}$ satisfies the strong Wolfe conditions (2.8) and (2.10). Then the following hold :

(i) The method with $\beta_{k}=\beta_{k}^{P R+}$ and $p_{k}=y_{k-1}$ (or $p_{k}=g_{k}$ ) converges in the sense that $\liminf _{k \rightarrow \infty}\left\|g_{k}\right\|=0$.

(ii) The method with $\beta_{k}=\beta_{k}^{H S+} \equiv \max \left\{\beta_{k}^{H S}\right.$, 0 0 and $p_{k}=y_{k-1}$ (or $p_{k}=g_{k}$ ) converges in the sense that $\liminf _{k \rightarrow \infty}\left\|g_{k}\right\|=0$.

Proof. In each case, since $\beta_{k} \geq 0$ holds, condition (C1) of Theorem 2.1 is satisfied. It suffices to prove that $(\mathrm{C} 2)$ holds in each case. Accordingly, we assume that there exists $\varepsilon$ such that $0<\varepsilon \leq\left\|g_{k}\right\|$ holds for all $k$.

(i) It follows from $\beta_{k}=\beta_{k}^{P R+}$ and $p_{k}=y_{k-1}$ that

$$
\begin{aligned}
\left|\psi_{k}\right| & =\left|\max \left\{\frac{g_{k}^{T} y_{k-1}}{\left\|g_{k-1}\right\|^{2}}, 0\right\}\left\|g_{k}\right\|\left\|y_{k-1}\right\|\left(g_{k}^{T} y_{k-1}\right)^{\dagger}\right| \\
& \leq \frac{\left\|g_{k}\right\|\left\|y_{k-1}\right\|}{\left\|g_{k-1}\right\|^{2}} \\
& \leq \frac{2 L \widehat{\gamma} \hat{a}}{\varepsilon^{2}}=\bar{b} .
\end{aligned}
$$

If $\bar{b}$ is not greater than 1 , define $b=1+\bar{b}$, so that $b>1$ and $b \geq \bar{b}$, else define $b=\bar{b}$. Now, we define $\xi=\varepsilon^{2} /(L \widehat{\gamma} b)$. If $\left\|s_{k-1}\right\| \leq \xi$, we have

$$
\left|\psi_{k}\right| \leq \frac{L \widehat{\gamma}\left\|s_{k-1}\right\|}{\varepsilon^{2}} \leq \frac{1}{b}
$$

which implies that Property A holds.

Next we consider the case of $\beta_{k}=\beta_{k}^{P R+}$ and $p_{k}=g_{k}$. Then we have

$$
\left|\psi_{k}\right|=\left|\max \left\{\frac{g_{k}^{T} y_{k-1}}{\left\|g_{k-1}\right\|^{2}}, 0\right\}\right| \leq \frac{\left\|g_{k}\right\|\left\|y_{k-1}\right\|}{\left\|g_{k-1}\right\|^{2}}
$$


and hence we can prove that Property A holds for the case $p_{k}=g_{k}$ in the same way as for the case $p_{k}=y_{k-1}$. Therefore the proof of (i) is complete.

(ii) It follows from $\beta_{k}=\beta_{k}^{H S+}, p_{k}=y_{k-1}$ and (2.10) that

$$
\begin{aligned}
\left|\psi_{k}\right| & =\left|\max \left\{\frac{g_{k}^{T} y_{k-1}}{d_{k-1}^{T} y_{k-1}}, 0\right\}\left\|g_{k}\right\|\left\|y_{k-1}\right\|\left(g_{k}^{T} y_{k-1}\right)^{\dagger}\right| \\
& \leq \frac{\left\|g_{k}\right\|\left\|y_{k-1}\right\|}{(1-\sigma)\left\|g_{k-1}\right\|^{2}} \\
& \leq \frac{2 L \widehat{\gamma} \hat{a}}{(1-\sigma) \varepsilon^{2}}=\bar{b} .
\end{aligned}
$$

If $\bar{b}$ is not greater than 1 , define $b=1+\bar{b}$, so that $b>1$ and $b \geq \bar{b}$, else define $b=\bar{b}$. Now, we define $\xi=(1-\sigma) \varepsilon^{2} /(L \widehat{\gamma} b)$. If $\left\|s_{k-1}\right\| \leq \xi$, we have

$$
\left|\psi_{k}\right| \leq \frac{L \widehat{\gamma}\left\|s_{k-1}\right\|}{(1-\sigma) \varepsilon^{2}} \leq \frac{1}{b}
$$

which implies that Property A holds.

Next we consider the case of $\beta_{k}=\beta_{k}^{H S+}$ and $p_{k}=g_{k}$. Then we have

$$
\left|\psi_{k}\right|=\left|\max \left\{\frac{g_{k}^{T} y_{k-1}}{d_{k-1}^{T} y_{k-1}}, 0\right\}\right| \leq \frac{\left\|g_{k}\right\|\left\|y_{k-1}\right\|}{(1-\sigma)\left\|g_{k-1}\right\|^{2}},
$$

and hence we can prove that Property $\mathrm{A}$ holds for the case $p_{k}=g_{k}$ in the same way as for the case $p_{k}=y_{k-1}$. Therefore the proof of (ii) is complete.

\section{Three-term conjugate gradient method based on multi-step quasi-Newton method}

In this section, we propose a three-term conjugate gradient method based on the multistep quasi-Newton method. In order to introduce a new choice of $\beta_{k}$ and $p_{k}$, let us briefly refer to the multi-step quasi-Newton method by Ford and Moghrabi [7,8]. The search direction $d_{k}$ of their method is given by $d_{k}=-H_{k} g_{k}$, where $H_{k}$ approximates the inverse Hessian of the objective function and it is updated by the multi-step BFGS formula:

$$
H_{k}=\left(I-\frac{\widehat{w}_{k-1} \widehat{r}_{k-1}^{T}}{\widehat{r}_{k-1}^{T} \widehat{w}_{k-1}}\right)^{T} H_{k-1}\left(I-\frac{\widehat{w}_{k-1} \widehat{r}_{k-1}^{T}}{\widehat{r}_{k-1}^{T} \widehat{w}_{k-1}}\right)+\frac{\widehat{r}_{k-1} \widehat{r}_{k-1}^{T}}{\widehat{r}_{k-1}^{T} \widehat{w}_{k-1}}
$$

and

$$
\widehat{r}_{k-1}=s_{k-1}-\widehat{\phi}_{k} s_{k-2}, \quad \widehat{w}_{k-1}=y_{k-1}-\widehat{\phi}_{k} y_{k-2} \quad \text { and } \quad \widehat{\phi}_{k}=\frac{g_{k}^{T} s_{k-1}}{g_{k}^{T} s_{k-2}} .
$$

Incorporating a parameter $t_{k} \geq 0$ into $\widehat{w}_{k}$, we redefine

$$
\widehat{w}_{k-1}=y_{k-1}-t_{k} \widehat{\phi}_{k} y_{k-2} \text {. }
$$


If $H_{k-1} \equiv I$, then the above multi-step BFGS method becomes the multi-step limitedmemory BFGS method, where the memory equals 1 . Since $g_{k}^{T} \widehat{r}_{k-1}=0$, the search direction $d_{k}$ is given by

$$
\begin{aligned}
d_{k} & =-\left(I-\frac{\widehat{w}_{k-1} \widehat{r}_{k-1}^{T}}{\widehat{r}_{k-1}^{T} \widehat{w}_{k-1}}\right)^{T}\left(I-\frac{\widehat{w}_{k-1} \widehat{r}_{k-1}^{T}}{\widehat{r}_{k-1}^{T} \widehat{w}_{k-1}}\right) g_{k}-\frac{\widehat{r}_{k-1} \widehat{r}_{k-1}^{T}}{\widehat{r}_{k-1}^{T} \widehat{w}_{k-1}} g_{k} \\
& =-g_{k}+\frac{g_{k}^{T} \widehat{w}_{k-1}}{\widehat{r}_{k-1}^{T} \widehat{w}_{k-1}} \widehat{r}_{k-1} .
\end{aligned}
$$

This search direction can be rewritten as the form:

$$
d_{k}=-g_{k}+\beta_{k}^{M S} d_{k-1}-\beta_{k}^{M S} \phi_{k} d_{k-2}
$$

where

$$
\begin{aligned}
\phi_{k} & =\frac{g_{k}^{T} d_{k-1}}{g_{k}^{T} d_{k-2}} \\
r_{k-1} & =d_{k-1}-\phi_{k} d_{k-2} \\
w_{k-1} & =y_{k-1}-t_{k} \frac{\alpha_{k-1}}{\alpha_{k-2}} \phi_{k} y_{k-2}
\end{aligned}
$$

and

$$
\beta_{k}^{M S}=\frac{g_{k}^{T} w_{k-1}}{r_{k-1}^{T} w_{k-1}} .
$$

Since (3.2) cannot be defined for the case $g_{k}^{T} d_{k-2}=0$, we replace (3.2) with

$$
\phi_{k}=g_{k}^{T} d_{k-1}\left(g_{k}^{T} d_{k-2}\right)^{\dagger}
$$

as a safeguard, and by considering (2.2), the direction (3.1) can be rewritten by

$$
d_{k}=-g_{k}+\beta_{k}^{M S}\left(g_{k}^{T} d_{k-2}\right)^{\dagger}\left\{\left(g_{k}^{T} d_{k-2}\right) d_{k-1}-\left(g_{k}^{T} d_{k-1}\right) d_{k-2}\right\} .
$$

We note that this corresponds to the three-term conjugate gradient method (2.2) with $p_{k}=d_{k-2}$ and $\beta_{k}=\beta_{k}^{M S}$. In addition, in order to establish the global convergence of our method, we modify (3.5) as follows:

$$
\beta_{k}^{M S+}=\max \left\{\frac{g_{k}^{T} w_{k-1}}{r_{k-1}^{T} w_{k-1}}, 0\right\} .
$$

If we use the exact line search, then $\phi_{k}=0$ and $\beta_{k}^{M S+}=\max \left\{g_{k}^{T} y_{k-1} / d_{k-1}^{T} y_{k-1}, 0\right\}$, and hence our method reduces to a modified HS $(\mathrm{HS}+)$ method.

Now we consider the global convergence of the proposed method. For this purpose, we make the following additional assumptions.

\section{Assumption 3.1.}


1. Assume that there exists a positive constant $\tau_{1}$ such that, for all $k$,

$$
\left\|g_{k}\right\|\left\|d_{k-2}\right\|\left|g_{k}^{T} d_{k-2}\right|^{\dagger} \leq \tau_{1}
$$

2. Assume that there exists a positive constant $\tau_{2}$ such that, for all $k$,

$$
\left|g_{k-1}^{T} r_{k-1}\right| \geq \tau_{2}\left|g_{k-1}^{T} d_{k-1}\right|
$$

3. Assume that there exists a constant $\tau_{3}$ that satisfies $0 \leq \tau_{3}<1$ and

$$
t_{k} \frac{\alpha_{k-1}}{\alpha_{k-2}}\left|\phi_{k}\right| \leq \tau_{3} \min \left\{\left|g_{k}^{T} y_{k-1}\right|\left|g_{k}^{T} y_{k-2}\right|^{\dagger},\left|r_{k-1}^{T} y_{k-1}\right|\left|r_{k-1}^{T} y_{k-2}\right|^{\dagger}\right\} \quad \text { for all } k
$$

Using Theorem 2.1, we obtain the following global convergence property.

Theorem 3.1. Suppose that Assumptions 2.1 and 3.1 are satisfied. Consider the method (2.1)-(2.2) with (3.8) and $p_{k}=d_{k-2}$. Assume that $\alpha_{k}$ satisfies the strong Wolfe conditions (2.8) and (2.10). Then the method converges in the sense that $\liminf _{k \rightarrow \infty}\left\|g_{k}\right\|=0$.

Proof. By (3.8), $\beta_{k} \geq 0$ clearly holds. So we only prove that the proposed method satisfies condition $(\mathrm{C} 2)$ of Theorem 2.1. To this end, we assume that there exists a constant $\varepsilon>0$ such that

$$
\left\|g_{k}\right\| \geq \varepsilon \quad \text { for all } k \text {. }
$$

It follows from (3.4) and (3.11) that

$$
\begin{aligned}
\left|g_{k}^{T} w_{k-1}\right| & \leq\left|g_{k}^{T} y_{k-1}\right|+t_{k} \frac{\alpha_{k-1}}{\alpha_{k-2}}\left|\phi_{k} g_{k}^{T} y_{k-2}\right| \\
& \leq\left(1+\tau_{3}\right)\left|g_{k}^{T} y_{k-1}\right| \\
& \leq\left(1+\tau_{3}\right) L\left\|g_{k}\right\|\left\|s_{k-1}\right\| .
\end{aligned}
$$

By (3.4), (3.11) and the fact $g_{k}^{T} r_{k-1}=0$, we have

$$
\begin{aligned}
\left|r_{k-1}^{T} w_{k-1}\right| & \geq\left|r_{k-1}^{T} y_{k-1}\right|-t_{k} \frac{\alpha_{k-1}}{\alpha_{k-2}}\left|\phi_{k} r_{k-1}^{T} y_{k-2}\right| \\
& \geq\left(1-\tau_{3}\right)\left|r_{k-1}^{T} y_{k-1}\right| \\
& =\left(1-\tau_{3}\right)\left|g_{k-1}^{T} r_{k-1}\right| .
\end{aligned}
$$

It follows from (3.10) and (2.3) that

$$
\left|g_{k-1}^{T} r_{k-1}\right| \geq \tau_{2}\left|g_{k-1}^{T} d_{k-1}\right|=\tau_{2}\left\|g_{k-1}\right\|^{2}
$$

Therefore (3.13) yields

$$
\left|r_{k-1}^{T} w_{k-1}\right| \geq \tau_{2}\left(1-\tau_{3}\right)\left\|g_{k-1}\right\|^{2}
$$


By (3.8), (3.12) and (3.14), we have

$$
\begin{aligned}
\beta_{k}^{M S+} & \leq \frac{\left|g_{k}^{T} w_{k-1}\right|}{\left|r_{k-1}^{T} w_{k-1}\right|} \\
& \leq \frac{\left(1+\tau_{3}\right) L\left\|g_{k}\right\|\left\|s_{k-1}\right\|}{\tau_{2}\left(1-\tau_{3}\right)\left\|g_{k-1}\right\|^{2}} \\
& \leq \frac{\left(1+\tau_{3}\right) L \widehat{\gamma}\left\|s_{k-1}\right\|}{\tau_{2}\left(1-\tau_{3}\right) \varepsilon^{2}} .
\end{aligned}
$$

Since the choice $p_{k}=d_{k-2}$ in (2.2) and (2.15) yield

$$
\psi_{k}=\beta_{k}^{M S+}\left\|g_{k}\right\|\left\|p_{k}\right\|\left(g_{k}^{T} p_{k}\right)^{\dagger}=\beta_{k}^{M S+}\left\|g_{k}\right\|\left\|d_{k-2}\right\|\left(g_{k}^{T} d_{k-2}\right)^{\dagger},
$$

(3.15) and (3.9) give

$$
\begin{aligned}
\left|\psi_{k}\right| & \leq \frac{\tau_{1}\left(1+\tau_{3}\right) L \widehat{\gamma}\left\|s_{k-1}\right\|}{\tau_{2}\left(1-\tau_{3}\right) \varepsilon^{2}} \\
& \leq \frac{2 \tau_{1}\left(1+\tau_{3}\right) L \widehat{a} \widehat{\gamma}}{\tau_{2}\left(1-\tau_{3}\right) \varepsilon^{2}}=\bar{b}
\end{aligned}
$$

We define $b=1+\bar{b}$ and

$$
\xi=\frac{\tau_{2}\left(1-\tau_{3}\right) \varepsilon^{2}}{\tau_{1}\left(1+\tau_{3}\right) L \widehat{\gamma} b}
$$

Then, if $\left\|s_{k-1}\right\| \leq \xi$, we have

$$
\left|\psi_{k}\right| \leq \frac{\tau_{1}\left(1+\tau_{3}\right) L \widehat{\gamma} \xi}{\tau_{2}\left(1-\tau_{3}\right) \varepsilon^{2}} \leq \frac{1}{b} .
$$

Therefore, Property A holds. Thus from Theorem 2.1, the theorem is true.

If $g_{k}^{T} d_{k-2}$ equals zero infinitely many times, the search direction becomes the steepest descent direction infinitely many times, which implies that $\lim _{\inf _{k \rightarrow \infty}}\left\|g_{k}\right\|=0$. So it is sufficient to consider the case $g_{k}^{T} d_{k-2} \neq 0$ for all $k$ sufficiently large. We note that assumption (3.9) yields

$$
\left|g_{k-1}^{T} r_{k-1}\right| \geq\left|g_{k-1}^{T} d_{k-1}\right|-\left|\phi_{k}\right|\left|g_{k-1}^{T} d_{k-2}\right| \geq\left(1-\frac{\tau_{1} \sigma^{2}\left\|g_{k-2}\right\|^{2}}{\left\|g _ { k } \left|\|\mid\| d_{k-2} \|\right.\right.}\right)\left|g_{k-1}^{T} d_{k-1}\right| .
$$

If $\sigma$ is chosen to be sufficiently small and $\frac{\left\|g_{k-2}\right\|^{2}}{\left\|g_{k}\right\|\left\|d_{k-2}\right\|}$ is bounded, then (3.10) holds. If $\frac{\left\|g_{k-2}\right\|^{2}}{\left\|g_{k}\right\|\left\|d_{k-2}\right\|}$ is unbounded, then $\liminf _{k \rightarrow \infty}\left\|g_{k}\right\|\left\|d_{k-2}\right\|=0$ holds from (2.7), and it implies $\liminf _{k \rightarrow \infty}\left\|g_{k}\right\|=0$ or $\liminf _{k \rightarrow \infty}\left\|d_{k}\right\|=0$. By Lemma 2.2, $\liminf _{k \rightarrow \infty}\left\|d_{k}\right\|=0$ leads $\liminf _{k \rightarrow \infty}\left\|g_{k}\right\|=0$, which is the desired result. Thus if (3.9) holds, then assumption (3.10) is not unreasonable. In our numerical experiments of Section 4, if (3.9) with $\tau_{1}=10^{15}$ does not hold, then we use the steepest descent direction. However, such a case did not occur in our numerical results. 


\section{Numerical results}

In this section, we report some numerical results. We investigated numerical performance of the proposed algorithms on 79 problems in the CUTEr $[1,11]$ library. Except for 8 problems, we used the default value of parameter included in each problem. Dimensions of the test problems lay on the range from 2 to 10000. We examined the following methods, where we denote CG and 3TCG by conjugate gradient methods and three-term conjugate gradient methods, respectively:

1. CG-DESCENT : CG by Hager and Zhang $[12,14]$

2. HS : CG with $\beta_{k}=\beta^{H S}$

3. $\mathrm{PR}+\quad$ : $\mathrm{CG}$ with $\beta_{k}=\beta^{P R+}$

4. FR : CG with $\beta_{k}=\beta^{F R}$

5. DY : CG with $\beta_{k}=\beta^{D Y}$

6. $3 \mathrm{HS}+\quad: 3 \mathrm{TCG}$ with $\beta_{k}=\beta^{H S+}$ and $p_{k}=y_{k-1}$

7. $3 \mathrm{PR}+\quad: 3 \mathrm{TCG}$ with $\beta_{k}=\beta^{P R+}$ and $p_{k}=y_{k-1}$

8. $3 \mathrm{MS}+\quad$ : $3 \mathrm{TCG}$ with $\beta_{k}=\beta^{M S+}, p_{k}=d_{k-2}$ and $t_{k}=1$.

In order to compare three-term conjugate gradient methods with conjugate gradient methods, we coded HS, PR+, FR, DY, 3HS+, 3PR + and 3MS+ by using the software package CG-DESCENT developed by Hager and Zhang [12,14], in which the line search and parameters were set as default. Since CG methods except for CG-DESCENT do not generally generate a descent search direction, we restart as the direction of steepest descent when a descent search direction is not produced. As stated in Section 3, for 3MS+, if $\left\|g_{k}\right\|\left\|d_{k-2}\right\|\left|g_{k}^{T} d_{k-2}\right|^{\dagger} \leq 10^{15}$, then we use the restart technique. However, such a case did not occur in our numerical experiments. We recognize that these numerical experiments are against $3 \mathrm{HS}+, 3 \mathrm{PR}+$ and $3 \mathrm{MS}+$, because the code CG-DESCENT is suitably tuned to the CG method by Hager and Zhang. Computational costs of $3 \mathrm{HS}+, 3 \mathrm{PR}+$ and 3MS+ may be reduced by effectively tuning the code, but it is beyond the scope of this paper. In the line search, we used the Wolfe conditions (2.8) and (2.9). Although we also tested $3 \mathrm{HS}+, 3 \mathrm{PR}+$ and $3 \mathrm{MS}+$ with the strong Wolfe conditions (2.8) and (2.10) for some problems, the results are not so different from results of the methods using the Wolfe conditions.

As stated in Section 2, if $g_{k}^{T} y_{k-1} \neq 0$, the search directions of 3HS+ and 3PR + become those given by Zhang et al. [19,21]. However their line search is not same as ours, and hence $3 \mathrm{HS}+$ and $3 \mathrm{PR}+$ are different from the algorithms by Zhang et al.

The stopping condition was

$$
\left\|g_{k}\right\|_{1} \leq 10^{-6}
$$

We stopped the algorithm if CPU time exceeds 500(sec) or if a numerical overflow occurs while the method tries to compute $f\left(x_{k}+\alpha_{k} d_{k}\right)$. However the second case did not occur.

We adopt the performance profiles by Dolan and Moré [5] to compare the performance among the tested methods. Figure 1-4 are the performance profile measured by CPU time, 
the number of iterations, the number of function evaluations and the number of gradient evaluations, respectively. In Figure 1, CG-DESCENT performed well from the viewpoint of CPU time. Since the code was not tuned for our methods, there was a case where our methods needed more CPU time. For example, for small-scale problems, there are the cases that CPU time of CG-DESCENT is $0.01(\mathrm{sec})$ and CPU time of $3 \mathrm{MS}+$ is $0.02(\mathrm{sec})$, and hence the line of $3 \mathrm{MS}+$ in Figure 1 much goes up at $\tau=2$. Accordingly, the numerical performance should be compared by measures different from CPU time. This is a reason why we give Figures 2-4. In Figures 2-4, we see that CG-DESCENT also performed well, and $3 \mathrm{PR}+, 3 \mathrm{HS}+$ and $\mathrm{PR}+$ are comparable with CG-DESCENT. On the other hand, $3 \mathrm{MS}+$ is slightly outperformed by CG-DESCENT and is comparable with HS.

From our numerical experiments, we see that 3TCG (especially $3 \mathrm{PR}+$ and $3 \mathrm{HS}+$ ) performed as well as CG-DESCENT did. However, there is room to improve 3TCG. Especially, since the line search in CG-DESCENT is also tuned for CG by Hager and Zhang, we need to develop a suitable line search for 3TCG. It is our further work. 


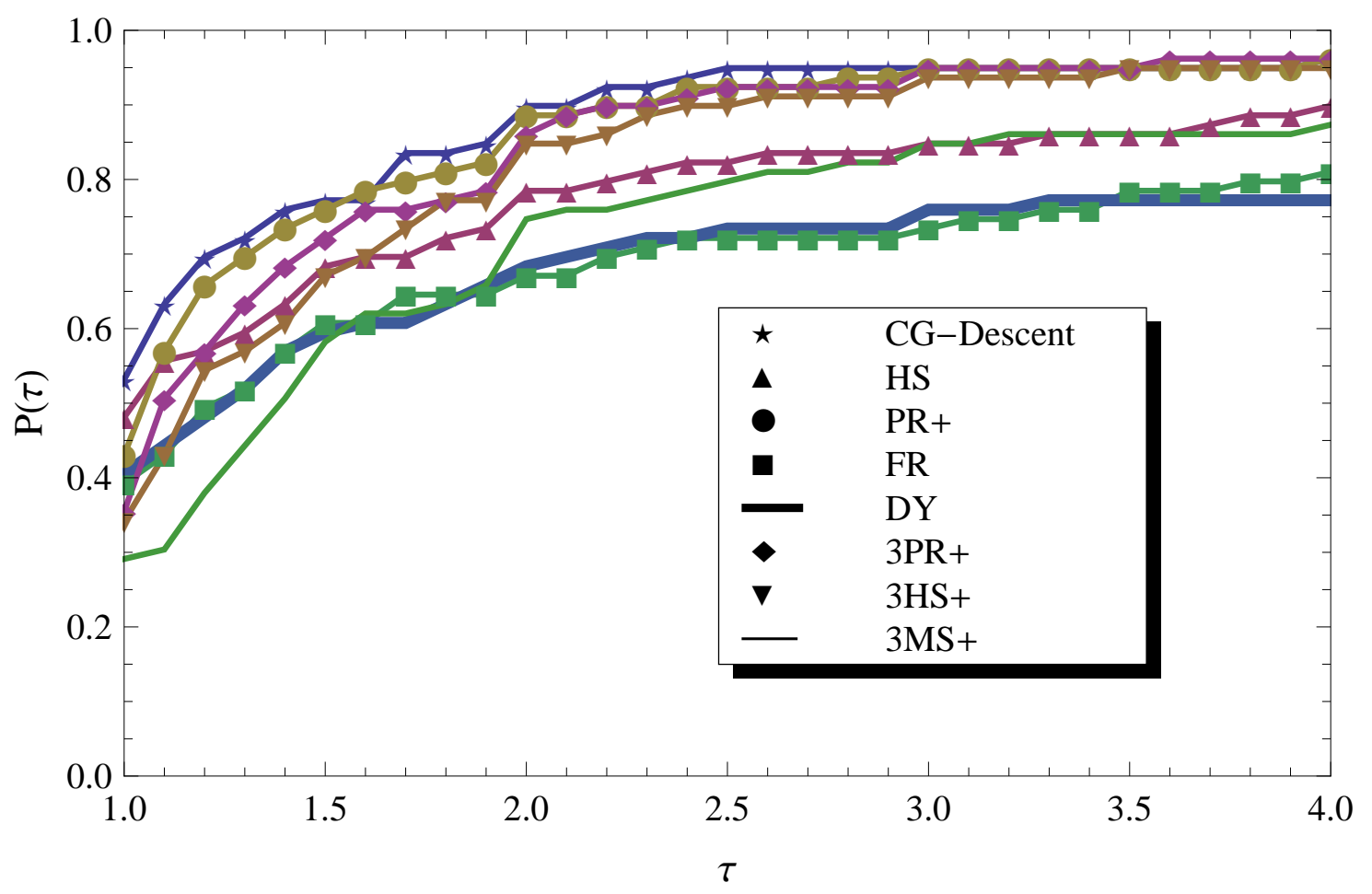

Figure 1: Performance profile by CPU time

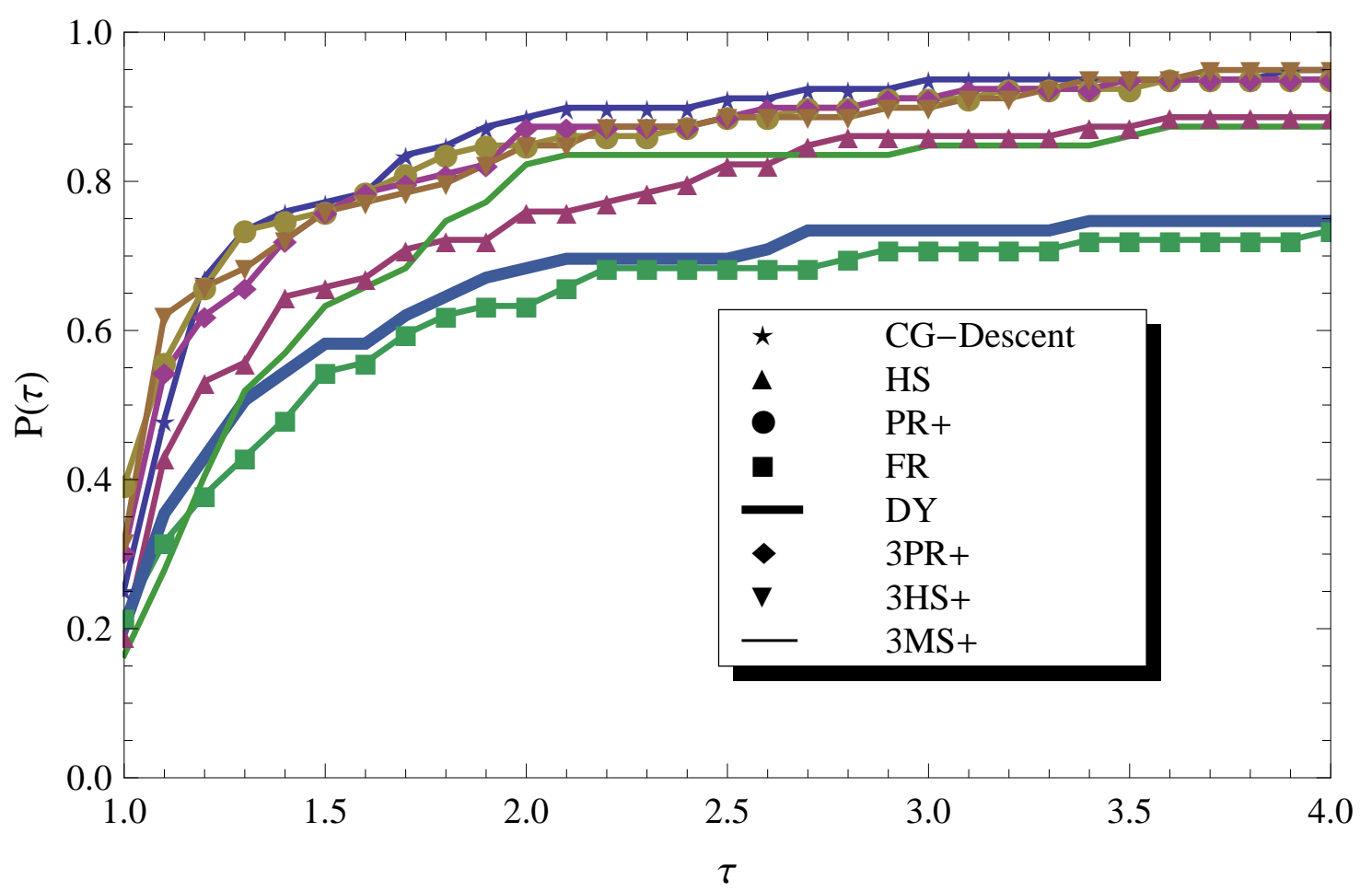

Figure 2: Performance profile by iterations 


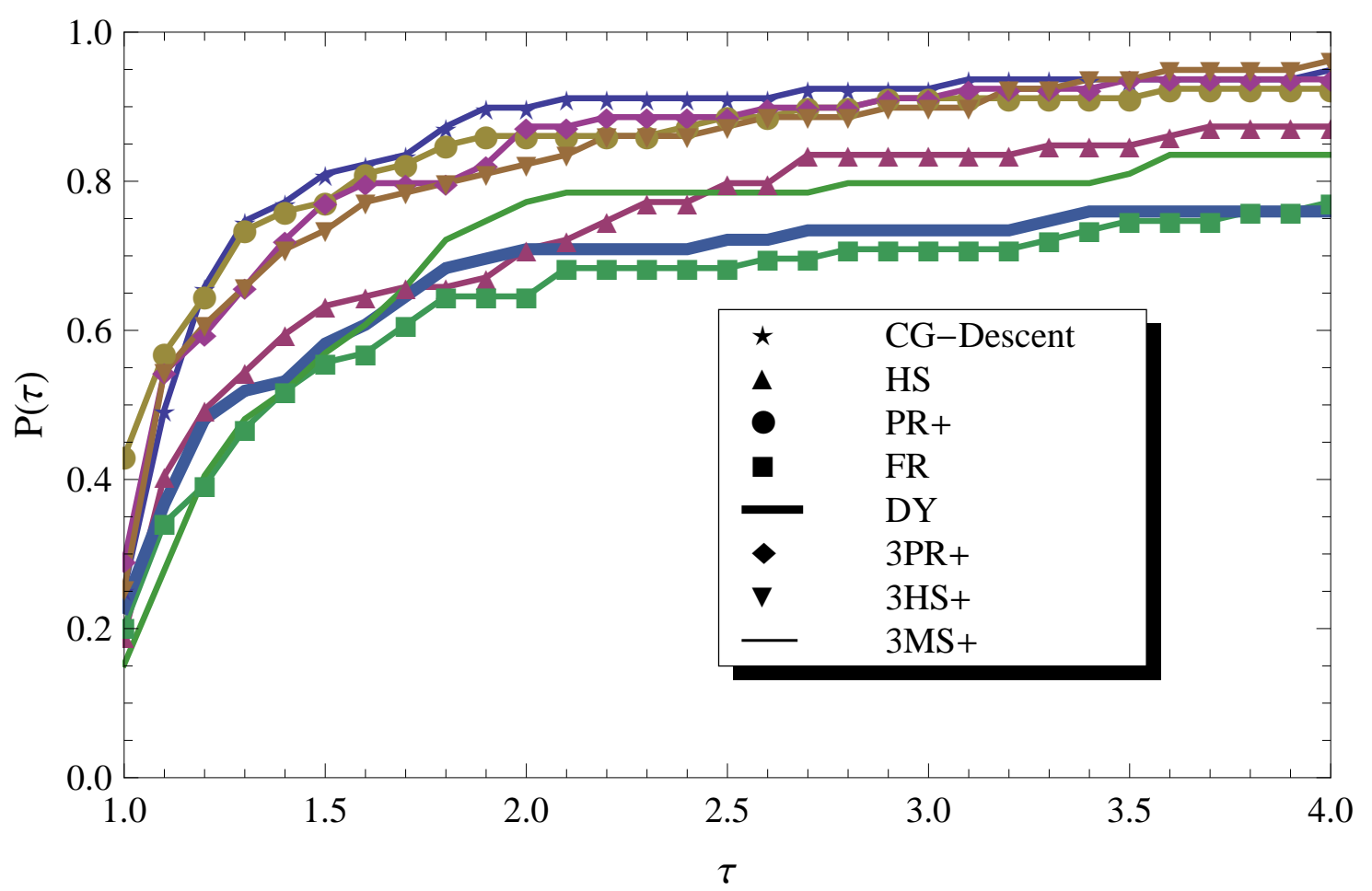

Figure 3: Performance profile by function evaluations

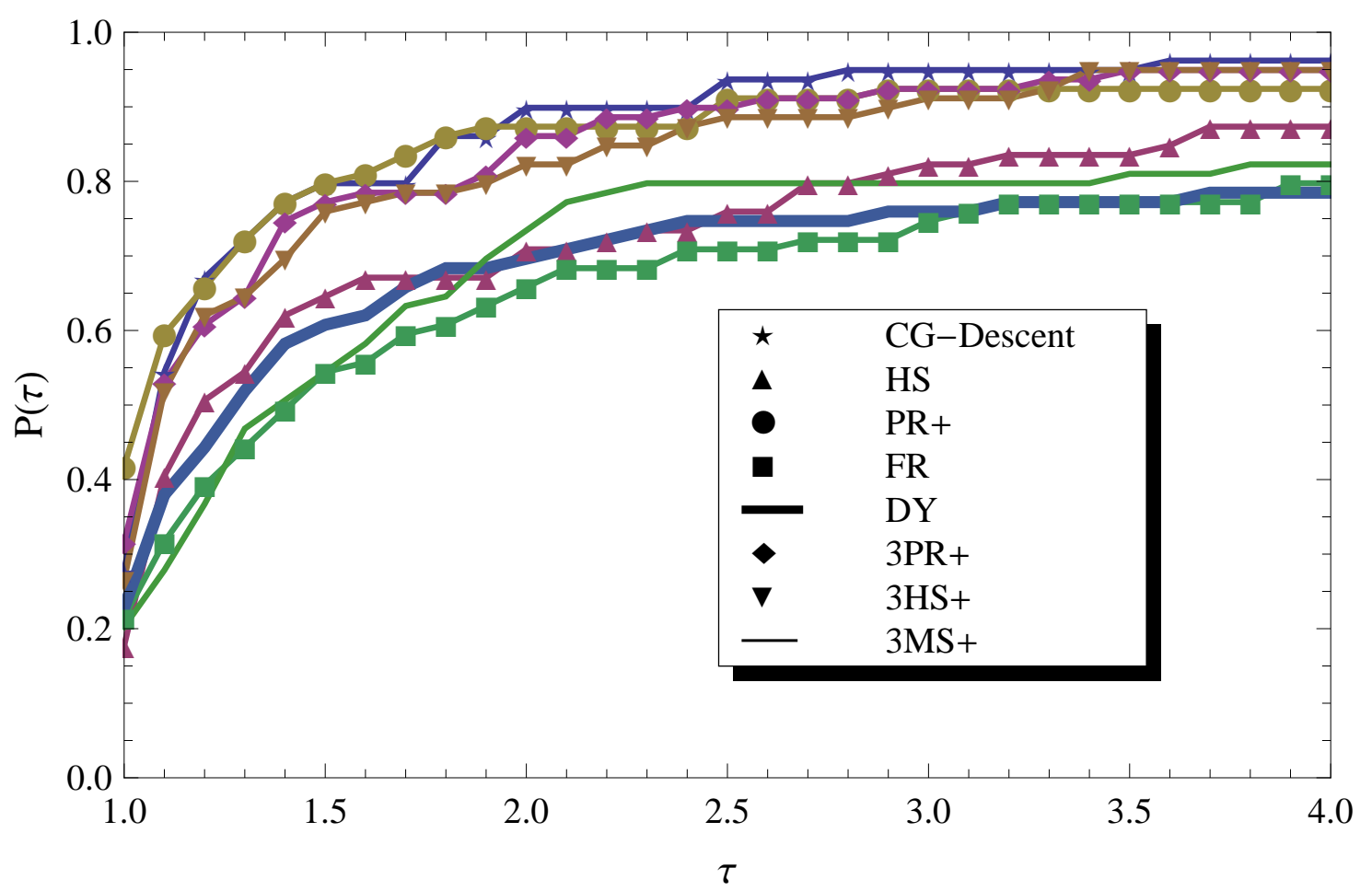

Figure 4: Performance profile by gradient evaluations 


\section{Conclusion}

In this paper, we have proposed a general form of three-term conjugate gradient methods which always satisfy the sufficient descent condition independently of line searches and a choice of $\beta_{k}$. Moreover, we have given a sufficient condition for the global convergence of the proposed method. We have also proposed a new three-term conjugate gradient method based on the multi-step quasi-Newton method as a specific method. We have given the numerical results of our method by using commonly used benchmark problems, and have shown that our method perform effectively. Our further works are to find a suitable choice of $p_{k}$ and to develop an efficient line search for three-term conjugate gradient methods.

\section{Acknowledgements}

The authors would like to thank the referees for valuable comments. The first and second authors are supported in part by the Grant-in-Aid for Scientific Research (C) 21510164 of Japan Society for the Promotion of Science.

\section{References}

[1] I. Bongartz, A.R. Conn, N.I.M. Gould and P.L. Toint, CUTE: constrained and unconstrained testing environments, ACM Transactions on Mathematical Software, 21 1995, $123-160$.

[2] W. Cheng, A two-term PRP-based descent method, Numerical Functional Analysis and Optimization, 28 (2007), 1217-1230.

[3] Y.H. Dai and L.Z. Liao, New conjugacy conditions and related nonlinear conjugate gradient methods, Applied Mathematics and Optimization, 43 (2001), 87-101.

[4] Y.H. Dai and Y. Yuan, A nonlinear conjugate gradient method with a strong global convergence property, SIAM Journal on Optimization, 10 (1999), 177-182.

[5] E.D. Dolan and J.J. Moré, Benchmarking optimization software with performance profiles, Mathematical Programming, 91 (2002), 201-213.

[6] R. Fletcher and C.M. Reeves, Function minimization by conjugate gradients, Computer Journal, 7 (1964), 149-154.

[7] J.A. Ford and I.A. Moghrabi, Alternative parameter choices for multi-step quasi-Newton methods, Optimization Methods and Software, 2 (1993), 357-370.

[8] J.A. Ford and I.A. Moghrabi, Multi-step quasi-Newton methods for optimization, Journal of Computational and Applied Mathematics, 50 (1994), 305-323.

[9] J.A. Ford, Y. Narushima and H. Yabe, Multi-step nonlinear conjugate gradient methods for unconstrained minimization, Computational Optimization and Applications, 40 (2008), $191-216$. 
[10] J.C. Gilbert and J. Nocedal, Global convergence properties of conjugate gradient methods for optimization, SIAM Journal on Optimization, 2 (1992), 21-42.

[11] N.I.M. Gould, D. Orban and P.L. Toint, CUTEr web site, http://cuter.rl.ac.uk/cuterwww/index.html.

[12] W.W. Hager and H. Zhang, A new conjugate gradient method with guaranteed descent and an efficient line search, SIAM Journal on Optimization, 16 (2005), 170-192.

[13] W.W. Hager and H. Zhang, A survey of nonlinear conjugate gradient methods, Pacific Journal of Optimization, 2 (2006), 35-58.

[14] W.W. Hager and H. Zhang, CG_DESCENT Version 1.4 User' Guide, University of Florida, November 2005, http://www.math.ufl.edu/ hager/.

[15] M.R. Hestenes and E. Stiefel, Methods of conjugate gradients for solving linear systems, Journal of Research of the National Bureau of Standards, 49 (1952), 409-436.

[16] J. Nocedal and S.J. Wright, Numerical Optimization (Second Edition), Springer Series in Operations Research, Springer Verlag, New York, 2006.

[17] H. Yabe and N. Sakaiwa, A new nonlinear conjugate gradient method for unconstrained optimization, Journal of the Operations Research Society of Japan, 48 (2005), 284-296.

[18] H. Yabe and M. Takano, Global convergence properties of nonlinear conjugate gradient methods with modified secant condition, Computational Optimization and Applications, 28 (2004), 203-225.

[19] L. Zhang, W. Zhou and D.H. Li, A descent modified Polak-Ribière-Polyak conjugate gradient method and its global convergence, IMA Journal of Numerical Analysis, 26 (2006), 629-640.

[20] L. Zhang, W. Zhou and D.H. Li, Global convergence of a modified Fletcher-Reeves conjugate gradient method with Armijo-type line search, Numerische Mathematik, 104 (2006), $561-572$.

[21] L. Zhang, W. Zhou and D.H. Li, Some descent three-term conjugate gradient methods and their global convergence, Optimization Methods and Software, 22 (2007), 697-711 .

[22] W. Zhou and L. Zhang, A nonlinear conjugate gradient method based on the MBFGS secant condition, Optimization Methods and Software, 21 (2006), 707-714. 